\title{
A randomized, blinded, controlled trial to assess sand fly mortality of fluralaner administered orally in dogs
}

\author{
Sonia Ares Gomez ${ }^{1 *} \mathbb{D}$, Javier Lucientes², Juan Antonio Castillo², Maria Paz Peris², Sarah Delacour², Paula Ortega ${ }^{2}$,
} Ronald-Vladimir Oropeza ${ }^{2}$ and Albert Picado ${ }^{1 *}$

\begin{abstract}
Background: Leishmania infantum is the parasite responsible for the disease in humans known as zoonotic visceral leishmaniasis (ZVL). Dogs are considered the main domestic reservoir of ZVL and sand flies are the proven vectors. The use of systemic insecticides in dogs has been studied as an alternative strategy to control ZVL in endemic areas. One systemic insecticide in dogs, fluralaner, has a proven anti-sand fly effect in membrane-fed studies. However, the efficacy and duration on sand flies directly feeding from dogs treated with fluralaner remains unknown.

Methods: Direct feeding bioassays were performed on 10 beagle dogs that had been randomly assigned to two groups: one with five dogs orally treated with Bravecto ${ }^{\circledast}$ (fluralaner) and other five as a control. About 30 females of Phlebotomus papatasi were allowed to directly feed from dogs at seven days before the administration of the treatment and Days 3, 17, 31, 45 and 73 post-treatment. Sand fly mortality after feeding was observed every $24 \mathrm{~h}$ for 5 days. The Kaplan-Meyer method, Henderson-Tilton formula and a negative binomial mixed model were used to respectively calculate: (i) mortality and its 95\% confidence interval (Cl); (ii) efficacy of the insecticide at killing sand flies in $24 \mathrm{~h}$; and (iii) differences in the risk of sand fly death at $24 \mathrm{~h}$ after feeding.

Results: Control sand fly mortality $24 \mathrm{~h}$ after feeding was always $\leq 20 \%$ and mortality in the fluralaner group ranged from 2\% (95\% Cl: 0-4\%) 7 days before treatment to $100 \%$ at 3 days post-treatment. Fluralaner efficacy was 100, 93, 94 and $75 \%$ at Days $3,17,31$ and 45 , respectively $(P<0.0001)$. The increase in the risk of sand fly death was $32.9(95 \% \mathrm{Cl}$ : 4-263), 76 (95\% Cl: 8-705), 95.8 (95\% Cl: 9-1029) and 10.6 times (95\% Cl: 1.43-79) on Days 3, 17, 31 and 45, respectively

Conclusions: The efficacy of fluralaner, orally administered to dogs, against sand-flies was above $90 \%$ for 31 days. Fluralaner administered to dogs should be further evaluated as a control strategy in ZVL endemic areas.
\end{abstract}

Keywords: Zoonotic visceral leishmaniasis (ZVL), Sand flies, Dogs, Systemic insecticides, Fluralaner

\section{Background}

Fluralaner is a systemic insecticide from the ixosazoline group used for the control of ectoparasites in companion animals. Bravecto ${ }^{\circ}$ is the commercialized name of flavored chewable tablets of fluralaner $(25 \mathrm{mg} / \mathrm{kg}$ body weight) registered for dogs to control fleas and ticks infestations for 12 weeks [1]. Fluralaner belongs to the isoxazoline group. Isoxazolines act at the central nervous system or the neuromuscular junction of the insect blocking GABA-gated chloride channels, thus disrupting

\footnotetext{
* Correspondence: soniaares@gmail.com; albert.picado@isglobal.org

${ }^{1}$ ISGlobal, Hospital Clínic - Universitat de Barcelona, Barcelona, Spain Full list of author information is available at the end of the article
}

neuronal signaling and muscle regulation causing insect death $[2,3]$. Based on the mechanism of action, fluralaner and other systemic insecticides used in dogs may have an anti-sand fly effect $[4,5]$. The effect of fluralaner on sand fly mortality was demonstrated in a randomized clinical trial in dogs using membrane feeding bioassays [6]. This proof of concept study reported sand fly mortalities between $60-80 \%$ for 30 days in dogs treated with fluralaner [6]. However, the high sand fly mortality in the control group precluded accurately estimating the mortality caused by fluralaner. Membrane-feeding was also used to demonstrate that fluralaner, mixed with

(c) The Author(s). 2018 Open Access This article is distributed under the terms of the Creative Commons Attribution 4.0 International License (http://creativecommons.org/licenses/by/4.0/), which permits unrestricted use, distribution, and 
rabbit blood, was effective against different species of sand flies [5].

Some species of sand flies are the vectors of Leishmania infantum, the parasite causing zoonotic visceral leishmaniasis (ZVL). ZVL is a major public health concern in some countries and regions (e.g. 3000 cases with 10-19\% lethality in Brazil per year [7]). Dogs, which are also affected by the parasite, are the main reservoir and they are often targeted by the leishmaniasis control programs in endemic countries. In Brazil, $L$. infantum infected dogs are culled to reduce the source of infection [7]. This strategy is controversial and has had a limited impact on the number of ZVL cases in humans [8-10]. The use of insecticide-impregnated collars in dogs [11-13] has been evaluated as an alternative to dog culling. Community-wide use of insecticide-impregnated collars has been proven to reduce the risk of $L$. infantum infection in humans $[13,14]$ but its use at a regional or national level has been limited due to several factors, namely the price of collars, collar losses, collar removal by dogs owners and high percentage of unreachable stray dogs $[12,14,15]$. Other topical insecticides in dogs such as topical lotions of deltamethrin, permethrin and fenthion, have also been suggested in vector control $[16,17]$. The use of systemic insecticides in dogs has been proposed as a vector control tool in endemic regions. A modelling study showed that treating $80 \%$ of dogs with a systemic insecticide that induces a sand fly mortality over $65 \%$ for 7.4 months would reduce the risk of $L$. infantum infection in humans by $80 \%$ [18].

To the best of our knowledge, there are currently no systemic insecticides for dogs registered against sand flies. Fluralaner, which can be given orally, is registered for dogs against ticks and fleas and has a proven anti-sand fly effect [5], could be a good control tool for ZVL in endemic areas $[4,18]$ In fact, Miglianico et al. [5] suggested the repurposing of fluralaner so it can be used in humans to control leishmaniasis and other vector-borne diseases. To further evaluate the efficacy and duration of fluralaner administered to dogs against sand flies we performed a blinded, randomized clinical trial using direct-feeding bioassays.

\section{Methods}

\section{Study site and dogs}

A total of 10 beagles ( 5 males and 5 females) between 12 and 24 months-old and weighting between 11-13 kg were used. The dogs were obtained from a licensed vendor and acclimatized to the study facility for more than 40 days prior to the beginning of the study. Dogs were uniquely identified by a subcutaneous microchip. The dogs were housed in individual inside-outside kennels that conformed to accepted guidelines for animal welfare. Dogs were fed a commercial dry dog ration once a day, water was provided ad libitum, and received regular exercise and social interaction. At the beginning of the study all dogs were clinically healthy and not clinically pregnant as determined by a veterinarian. Dogs had not been treated with drugs, baths, shampoos, or pesticides within 4 weeks preceding the beginning of the study and were not treated during the course of the study other than with fluralaner in the treated group.

\section{Study design}

The study was designed to detect at least $65 \%$ insecticide efficacy of fluralaner on blood-fed Phlebotomus papatasi feeding on dogs, with a power of 0.8 and an alpha of 0.05 .

Two groups of 5 dogs were randomly selected blocking by gender. Once two groups were formed, one with 3 females and 2 males and other with 2 females and 3 males, treatment was randomly assigned to one of the groups. The random selection was done using the sample function in R [19].

At Day 0, the dogs in the treatment group received one chewable tablet for medium-sized dogs ( $>10$ to 20 $\mathrm{kg}$ ) of $\mathrm{Bravecto}^{\circ}$, equivalent to $500 \mathrm{mg}$ of fluralaner, at the time of feeding, as indicated in the label. Control dogs received their regular ration only.

The effect of Bravecto ${ }^{\circ}$ on sand flies was assessed using direct-feeding bioassays at Days 3, 17, 31, 45 and 73 . Additionally, a direct feeding bioassay was performed 7 days before the administration of the treatment (Day -7).

Phlebotomus papatasi females, two- to seven-days-old from a colony reared in the University of Zaragoza were used in the direct-feeding bioassays. The colony was established in 2012 with P. papatasi trapped in the province of Zaragoza (Spain). The colony was maintained at $27{ }^{\circ} \mathrm{C}, 80 \%$ relative humidity and photoperiod of $17: 7 \mathrm{~h}$ (light:dark) [20].

On the days of the bioassay, fasted dogs were sedated with $0.5 \mathrm{mg} / \mathrm{kg}$ of intramuscular dexmedetomidine (Dexdomitor ${ }^{\circ}$ ). Once the dog was sedated, the previously shaved inner part of the ear was exposed to the bite of about 30 P. papatasi females. To facilitate the feeding and the recovery of blood-fed sand flies during the direct feeding bioassays $[21,22]$ the sand flies were introduced in a round container $(11.5 \mathrm{~cm}$ diameter and $7.5 \mathrm{~cm}$ height) covered by a cotton net that allowed them to feed through (Fig. 1a). To keep the ear warm, one bottle of $250 \mathrm{ml}$ of normal saline previously warm to $37{ }^{\circ} \mathrm{C}$ was placed on top (Fig. 1b). One hour or at least $30 \mathrm{~min}$ later, when most of the females were engorged, the sand flies were removed. The dogs received one intramuscular injection of atipamezole $(0.25$ $\mathrm{mg} / \mathrm{kg}$, Antisedan ${ }^{\circ}$ ) to revert the anesthesia. 


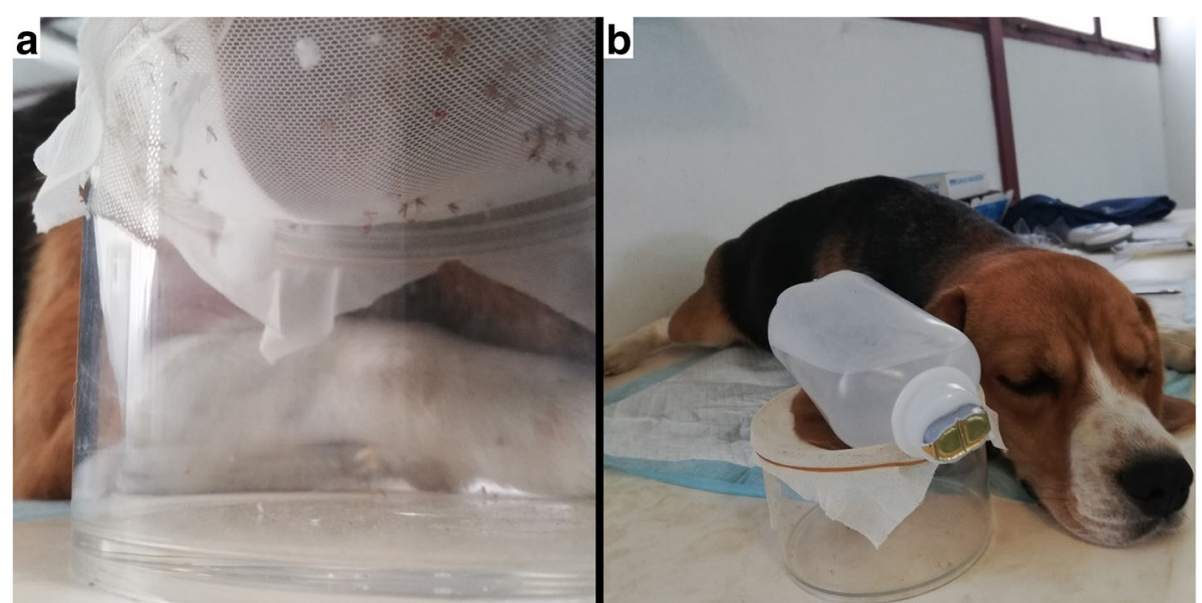

Fig. 1 Direct feeding bioassay of Phlebotomus papatasi directly feeding on a sedated dog. a Detail of the inner part of the ear exposed to the bite of about 30 P. papatasi females located inside a round container covered by a cotton net that allowed the sand flies to feed through. b Sedated dog with the ear exposed to sand flies being warmed by a bottle of $250 \mathrm{ml}$ of normal saline previously warm to $37^{\circ} \mathrm{C}$

For the ease of observation, engorged sand flies were separated in groups of five into a plastic cup of $90 \mathrm{ml}$ and $60 \mathrm{~mm}$ diameter with sucrose solution provided daily. The plastic cups were introduced into an incubator where humidity was controlled. Sand fly mortality was observed every $24 \mathrm{~h}$ during five days. Sand fly mortality and its $95 \%$ CI was calculated at each day for 5 days post-feeding using the Kaplan-Meyer method [23].

The researchers manipulating the sand flies and observing sand fly mortality were blind to treatment allocation.

\section{Statistical analysis}

Two methods were used to assess the sand fly mortality due to fluralaner. The Henderson-Tilton formula was used to estimate the efficacy of the insecticide at killing sand flies [24], and a negative binomial mixed model was used to estimate the risk of sand fly death due to fluralaner over time [25]. Both analyses used the sand fly mortality observed $24 \mathrm{~h}$ post-ingestion as the outcome.

Efficacy was measured as the percentage of sand fly mortality due to the insecticide effect and was calculated according to the following adapted Henderson-Tilton formula $[24,26]$ :

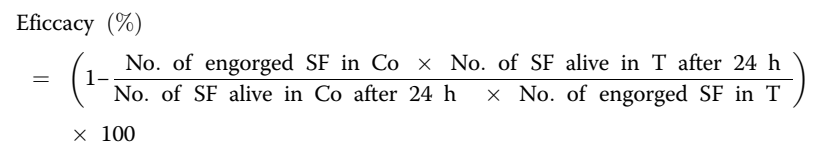

where $\mathrm{SF}$ is sand flies, Co is control group and $\mathrm{T}$ is treatment group. Fisher's exact test was used for each experimental day to test if differences in sand fly mortality were significantly different at alpha 0.05 . The European Medicine Agency uses as reference method a similar formula and efficacies above $80 \%$ should be achieved to demonstrate efficacy of ectoparasiticides [27].

In negative binomial mixed model the variable that identified each individual dog was included as random effect [25]. The model explanatory variables were treatment, day of study, and the interaction between treatment and Day. The interaction term allowed us to estimate differences in the sand fly mortality across time. The model dependent variable was the log of the number of sand flies dead $24 \mathrm{~h}$ post-feeding given the total number of engorged sand flies (offset). The exponential of the model estimates represents the incidence rate ratios (IRR) which indicate how many times the risk of death increases due to fluralaner.

All analyses were conducted in $\mathrm{R}$ (version 3.3) [19]. The MASS package [28] was used to conduct the negative binomial regression. The survival and coxme packages [29] were used to conduct the Kaplan-Meier table.

\section{Results}

The study was conducted between May and July 2018. No adverse effects related to the treatment were observed in any animal during the course of the study. A total of 909 and 849 specimens of P. papatasi were used in the treatment and control groups, respectively (Table 1 ). Control sand fly mortality $24 \mathrm{~h}$ after feeding was always $\leq 20 \%$ as established in the $\mathrm{EU}$ guidelines [27]. Kaplan-Meyer mortality at $24 \mathrm{~h}$ post-feeding (\%, and $95 \% \mathrm{CI}$ ) per group and bioassay are reported in Table 1; the data at 48, 72, 96 and $120 \mathrm{~h}$ post-feeding are provided in Additional file 1: Table S1 and the data for $24 \mathrm{~h}$ sand fly mortality per dog are provided in Additional file 1: Table S2. Sand fly mortality $24 \mathrm{~h}$ after feeding in the control group ranged from 1\% (95\% CI: 
Table 1 Observed efficacy and induced mortality of fluralaner against sand flies 24 hours after exposure on dogs

\begin{tabular}{|c|c|c|c|c|c|c|c|c|}
\hline \multirow[t]{2}{*}{ Direct feeding } & \multicolumn{3}{|c|}{ Control } & \multicolumn{5}{|c|}{ Fluralaner } \\
\hline & $\overline{n^{a}}$ & $\begin{array}{l}\text { Deaths }{ }^{\mathrm{b}} \\
n(\%)\end{array}$ & $95 \% \mathrm{Cl}$ & $\overline{N^{a}}$ & $\begin{array}{l}\text { Deaths }{ }^{b} \\
n(\%)\end{array}$ & $95 \% \mathrm{Cl}$ & $\begin{array}{l}\text { Efficacy } \\
(\%)\end{array}$ & $P$-value ${ }^{d}$ \\
\hline Day -7 & 138 & $3(2)$ & $0-5$ & 123 & $2(2)$ & $0-4$ & 0 & 0.75 \\
\hline Day 3 & 147 & $6(4)$ & $0-7$ & 151 & $151(100)$ & $n a^{e}$ & 100 & $<0.0001$ \\
\hline Day 17 & 150 & $3(2)$ & $0-4$ & 146 & $143(98)$ & $96-100$ & 93 & $<0.0001$ \\
\hline Day 31 & 148 & $2(1)$ & $0-3$ & 159 & $150(95)$ & $91-98$ & 94 & $<0.0001$ \\
\hline Day 45 & 141 & $13(10)$ & $5-15$ & 148 & $138(89)$ & 84-93 & 75 & $<0.0001$ \\
\hline Day 73 & 153 & $34(22)$ & $13-28$ & 182 & $75(42)$ & $35-49$ & 26 & $<0.08$ \\
\hline
\end{tabular}

${ }^{\mathrm{a}}$ Number of sand flies exposed to direct-feed on dogs

${ }^{\mathrm{b}}$ Number (\%) of sand flies dead $24 \mathrm{~h}$ after direct-feeding

'Efficacy as the percentage of phlebotomine mortality due to the insecticide effect calculated according to adapted Henderson-Tilton's formula

${ }^{d}$ (Two-sided) probability values associated with the comparison on sand fly mortality between control and fluralaner using Fisher's exact test

${ }^{\mathrm{e}}$ All samples showed $100 \%$ death so there is no $95 \% \mathrm{Cl}$

0-3\%) to $20 \%$ (95\% CI: $13-28 \%$ ) corresponding to Days 31 and 73 respectively. Mortality in the fluralaner group ranged from 2\% (95\% CI: 0-4\%) at Day -7 (before treatment) to $100 \%$ at Day 3 after treatment. At Days 17 and 31 the $24 \mathrm{~h}$ mortality and its $95 \% \mathrm{CI}$ were above 90\%. A decrease to $77 \%$ (95\% CI: 72-82\%) was observed at Day 45 and a further drop to $42 \%$ (95\% CI: $35-49 \%$ ) at Day 73 (Table 1).

Fluralaner showed efficacies of 100, 93 and $94 \%$ at Days 3, 17 and 31, respectively $(P<0.0001$; Table 1$)$. Between Days 45 (efficacy 75\%; $P<0.0001$ ) and 73 (29\%; $P=0.08$ ) the efficacy dropped below the threshold of $65 \%$ (Fig. 2).

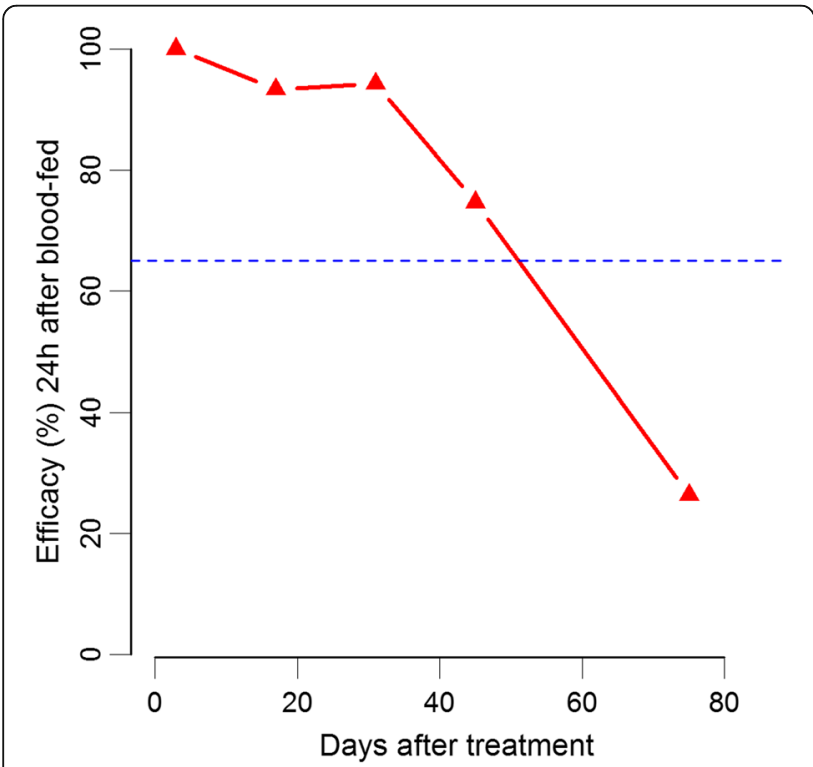

Fig. 2 Corrected efficacy (\%) according to Henderson-Tilton's

formula for Phlebotomus papatasi $24 \mathrm{~h}$ after feeding on dogs treated with one chewable tablet of Bravecto ${ }^{\oplus}$ on Days 3, 17, 31, 45 and 73 after treatment. The interrupted line indicates the efficacy threshold of $65 \%$
Fluralaner highly increased the risk of sand fly death (Table 2). The incidence rate ratio (IRR) was $32.9(95 \%$ CI: 4-263), 76 (95\% CI: 8-705), 95.8 (95\% CI: 9-1029) and 10.6 (95\% CI: 1.43-79) on Days 3, 17, 31 and 45, respectively (Table 2). The sand fly killing effect of fluralaner decreased after Day 31 post-treatment and on Day 73 the effect was not significant (Fig 3).

\section{Discussion}

One oral dose of Bravecto ${ }^{\circ}$ showed efficacies > $94 \%$ at killing sand flies for 31 days and $>75 \%$ for 45 days post-treatment. The sand fly risk of death $24 \mathrm{~h}$ after feeding increases more than 10 times when feeding from a treated dog and this effect was maintained for 45 days after treatment.

The efficacy of fluralaner administered to dogs at killing sand flies has already been demonstrated in a membrane-feeding study [6]. However, the mentioned study reported lower mortalities in the fluralaner group

Table 2 Negative binomial mixed model results including treatment group, experimental day and their interaction as fixed effects and dog as random effect. The incidence rate ratio (IRR) represents the increase in the rate of incidence compared with control per day

\begin{tabular}{llll}
\hline Explanatory variable $^{\mathrm{a}}$ & $\mathrm{IRR}^{\mathrm{b}}$ & $95 \% \mathrm{Cl}$ & $P$-value \\
\hline Day -7 & 0.75 & $0.09-4.85$ & 0.77 \\
Day 3 & 32.87 & $4.09-263$ & $0.001^{* *}$ \\
Day 17 & 76.06 & $8.20-705$ & $0.0002^{* *}$ \\
Day 31 & 95.85 & $8.92-1029$ & $0.0002^{* *}$ \\
Day 45 & 10.66 & $1.43-79$ & $0.02^{*}$ \\
Day 73 & 2.50 & $0.34-18$ & 0.36
\end{tabular}

${ }^{a}$ The explanatory variables used control group at each corresponding day after treatment as baseline comparison $(\operatorname{Pr}(>|z|))$

b Incidence rate ratio, as the ratio between the incidence rate in control group and the incidence rate for each treatment group, at the corresponding experimental day after treatment

*Significance level at $a=0.05$

**Significance level at $a=0.001$ 


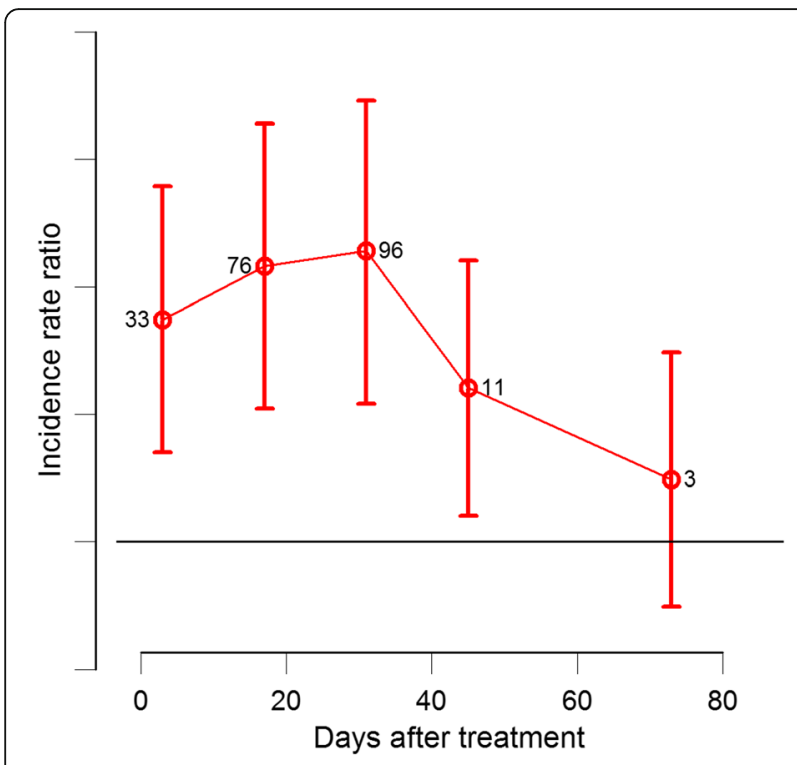

Fig. 3 Incidence rate ratios (IRR) for mortality of Phlebotomus papatasi $24 \mathrm{~h}$ after feeding on dogs treated with one chewable tablet of Bravecto ${ }^{\circledR}$ compared with control on Days 3, 17, 31, 45 and 73 after treatment. IRR was estimated from mixed negative binomial model were variable dog was included as a random effect, treatment, days after treatment, and their interaction were explanatory variables. The horizontal line marks the significant threshold IRR $=1$

$(42-80 \%)$ and higher mortalities in the control group (35-60\%). These discrepancies with our results could be due to the manipulation of the blood samples (freeze-thaw) made during the membrane-feeding study [30]. A recent laboratory study evaluated the $\mathrm{IC}_{50}$ (concentration at which $50 \%$ of the sand flies died $24 \mathrm{~h}$ after feeding) of fluralaner for two sand fly species, Lutzomyia longipalpis (vector of L. infantum in Latin America) and $P$. argentipes (vector of $L$. donovani in the Indian subcontinent). Lutzomyia longipalpis required twice the dose of fluralaner [655 ng/ $\mathrm{ml}$ (95\% CI: 537-796)] to kill 50\% of the sand flies compared to $P$. argentipes $[318 \mathrm{ng} / \mathrm{ml}(95 \% \mathrm{CI}: 278-$ 364)]. Bravecto ${ }^{\circ}$ demonstrated a plasma concentration of fluralaner maintained above $650 \mathrm{ng} / \mathrm{ml}$ for 40 days and above $350 \mathrm{ng} / \mathrm{ml}$, for 56 days post-treatment $[1,3,31]$. Our study found $P$. patatasi mortality above $75 \%$ for 45 days post-treatment. Phlebotomus papatasi has shown to be as susceptible to fluralaner as $P$. argentipes and more susceptible than $L$. longipalpis. In the Old World, P. perniciosus is the main vector of $L$. infantum, thus the experiment should have ideally been performed using a colony of $P$. perniciosus. However, the trial required a total of 1800 females and unfortunately the colony of $P$. perniciosus in the University of Zaragoza could not produce this number of sand flies; as such we had to use P. papatasi instead.
Based on a previous study we expect similar insecticide sensitivity between P. papatasi and P. perniciosus [32]. Because a higher concentration of fluralaner is needed to control L. longipalpis, a shorter period of action of Bravecto $^{\circ}$ could be expected in this sand fly species. Additionally, Bravecto ${ }^{\circ}$ administered to dogs also induced $100 \%$ triatomine mortality for 51 days [21].

\section{Conclusions}

A single chewable tablet of fluralaner was effective against sand flies feeding on treated dogs for 45 days (efficacy $>75 \%$ ). The community-wide use of fluralaner and other isoxazoline drugs registered for dogs such as afoxolaner or saloraner should be evaluated as a ZVL control strategy in endemic areas.

\section{Additional file}

Additional file 1: Table S1. Sand fly mortality, percentage, and 95\% Cl calculated using the Kaplan-Meier method from the mortality observed $24,48,72,96$ and $120 \mathrm{~h}$ after direct blood-feeding on dogs by treatment and sampling day. Table S2. Individual dog sand fly mortality and percentage observed $24 \mathrm{~h}$ after direct blood-feeding by treatment and sampling day. (DOCX $40 \mathrm{~kb}$ )

\section{Abbreviations}

Cl: Confidence interval; IRR: Incidence rate ratio; ZVL: Zoonotic visceral leishmaniasis

\section{Acknowledgements}

We thank Alberto Cortes who kindly collaborated in this project. ISGlobal is a member of the CERCA Programme, Generalitat de Catalunya.

\section{Funding}

This research was funded by the European Union's H2020 Programme under MSCA GA no. 642609.

\section{Availability of data and materials}

Data supporting the conclusions of this article are included within the article and its additional files. The datasets used and/or analyzed during the present study are available from the corresponding author upon reasonable request.

\section{Authors' contributions}

SAG, AP, JAC and JL designed the study protocol. SAG, PPP, SD and POH performed the direct feeding bioassays. PPP, SD and POH observed sand fly mortality. RVOV took care of the sand fly colony. SAG performed the statistical analysis. SAG and AP were major contributors in writing the manuscript. All authors read and approved the final manuscript.

\section{Ethics approval and consent to participate}

The study was conducted at the Faculty of Veterinary Medicine at the University of Zaragoza (Spain). Approvals for the animal experiments were obtained from the Universidad de Zaragoza (UNIZAR), Zaragoza, España (Comisión Ética asesora para la experimentación animal, ref. PI44/17) and the University of Barcelona, España, Comite Etic d'Experimentación Animal (CEEA no. 9/18). All animal handling and bioassay procedures, and the maintenance of the $P$. papatasi colony followed protocols approved by the ethical committee of the University of Zaragoza, Spain.

\section{Consent for publication}

Not applicable.

\section{Competing interests}

The authors declare that they have no competing interests. 


\section{Publisher's Note}

Springer Nature remains neutral with regard to jurisdictional claims in published maps and institutional affiliations.

\section{Author details}

'ISGlobal, Hospital Clínic - Universitat de Barcelona, Barcelona, Spain. ${ }^{2}$ Department of Animal Pathology, Faculty of Veterinary Medicine at the University of Zaragoza, Zaragoza, Spain.

Received: 17 September 2018 Accepted: 23 November 2018

Published online: 05 December 2018

\section{References}

1. Williams H, Young DR, Qureshi T, Zoller H, Heckeroth AR. Fluralaner, a novel isoxazoline, prevents flea (Ctenocephalides felis) reproduction in vitro and in a simulated home environment. Parasit Vectors. 2014;7:275.

2. Gassel M, Wolf C, Noack S, Williams H, Ilg T. The novel isoxazoline ectoparasiticide fluralaner: Selective inhibition of arthropod $\gamma$-aminobutyric acid- and I-glutamate-gated chloride channels and insecticidal/acaricidal activity. Insect Biochem Mol Biol. 2014;45:111-24.

3. Kilp S, Ramirez D, Allan MJ, Roepke RK, Nuernberger MC. Pharmacokinetics of fluralaner in dogs following a single oral or intravenous administration. Parasit Vectors. 2014;7:85.

4. Gomez SA, Picado A. Systemic insecticides used in dogs: potential candidates for phlebotomine vector control? Trop Med Int Health. 2017:22:755-64.

5. Miglianico M, Eldering M, Slater $H$, Ferguson $N$, Ambrose P, Lees RS, et al. Repurposing isoxazoline veterinary drugs for control of vector-borne human diseases. Proc Natl Acad Sci USA. 2018;115:E6920-6.

6. Gomez SA, Curdi JL, Hernandez JAC, Peris PP, Gil AE, Velasquez RVO, et al. Phlebotomine mortality effect of systemic insecticides administered to dogs. Parasit Vectors. 2018;11:230

7. Ministry of Health of Brazil. Manual de Vigilância e Controle da Leishmaniose Visceral. 1st ed. Brazil: (Brasilia -DF): Ministério da Saúde; 2014. p. 1-114.

8. Nunes CM, de LVMF, de PHB, Perri SHV, de AAM, Dias FEF, et al. Dog culling and replacement in an area endemic for visceral leishmaniasis in Brazil. Vet Parasitol. 2008;153:19-23.

9. Courtenay O, Quinnell RJ, Garcez LM, Shaw JJ, Dye C. Infectiousness in a cohort of Brazilian dogs: why culling fails to control visceral leishmaniasis in areas of high transmission. J Infect Dis. 2002;186:1314-20.

10. Barreto ML, Teixeira MG, Bastos Fl, Ximenes RA, Barata RB, Rodrigues LC. Successes and failures in the control of infectious diseases in Brazil: social and environmental context, policies, interventions, and research needs. Lancet. 2011;377:1877-89.

11. RA e S, de AAJ, Quint BB, Raffoul GES, Werneck GL, Rangel EF, et al. Effectiveness of dog collars impregnated with $4 \%$ deltamethrin in controlling visceral leishmaniasis in Lutzomyia longipalpis (Diptera: Psychodidade: Phlebotominae) populations. Mem Inst Oswaldo Cruz. 2018;113:e170377.

12. Leite BMM, Solcà M d S, Santos LCS, Coelho LB, Amorim LDAF, Donato LE, et al. The mass use of deltamethrin collars to control and prevent canine visceral leishmaniasis: a field effectiveness study in a highly endemic area. PLoS Negl Trop Dis. 2018;12:e0006496.

13. Camargo-Neves V, Rodas L, Calemes E. Cost effectiveness of deltamethrin impregnated collars (Scalibor) for the control of visceral leishmaniasis in human and canine populations in Brazil. Proceedings of the Second International Congress on Canine Leishmaniasis; 2010. p. 118-20.

14. Gavgani ASM, Hodjati MH, Mohite H, Davies CR. Effect of insecticideimpregnated dog collars on incidence of zoonotic visceral leishmaniasis in Iranian children: a matched-cluster randomised trial. Lancet. 2002:360:374-9.

15. Reithinger R, Coleman PG, Alexander B, Vieira EP, Assis G, Davies CR. Are insecticide-impregnated dog collars a feasible alternative to dog culling as a strategy for controlling canine visceral leishmaniasis in Brazil? Int J Parasitol. 2004;34:55-62

16. Courtenay O, Kovacic V, Gomes PA, Garcez LM, Quinnell RJ. A long-lasting topical deltamethrin treatment to protect dogs against visceral leishmaniasis. Med Vet Entomol. 2009;23:245-56.

17. Reithinger $R$, Teodoro $U$, Davies $C R$. Topical insecticide treatments to protect dogs from sand fly vectors of leishmaniasis. Emerg Infect Dis. 2001;7:872-6.
18. Gomez SA, Chapman LAC, Dilger E, Courtenay O, Picado A. Estimating the efficacy of community-wide use of systemic insecticides in dogs to control zoonotic visceral leishmaniasis: a modelling study in a Brazilian scenario. PLoS Negl Trop Dis. 2018;12:e0066797.

19. R Development Core Team. R: A language and environment for statistical computing. Vienna: R Foundation for Statistical Computing; 2015.

20. Molina R, Jimenez M, Alvar J. Methods in Sand Fly Research. 1st ed. Madrid, Spain: Servicio de Publicaciones Universidad de Alcalá de Henares; 2017.

21. Loza A, Talaga A, Herbas G, Canaviri RJ, Cahuasiri T, Luck L, et al. Systemic insecticide treatment of the canine reservoir of Trypanosoma cruzi induces high levels of lethality in Triatoma infestans, a principal vector of Chagas disease. Parasit Vectors. 2017;10:344.

22. Poché RM, Garlapati R, Singh MI, Poché DM. Evaluation of fipronil oral dosing to cattle for control of adult and larval sand flies under controlled conditions. J Med Entomol. 2013:50:833-7.

23. Ma ZS. Survival analysis approach to insect life table analysis and hypothesis testing: with particular reference to Russian wheat aphid (Diuraphis noxia (Mordvilko)) populations. Bull Entomol Res. 2010;100:315-24.

24. Henderson CF, Tilton EW. Tests with acaricides against the brown wheat mite. J Econ Entomol. 1955;48:157-61.

25. Alexander N. Analysis of parasite and other skewed counts. Trop Med Int Health. 2012;17:684-93.

26. Committee for Medicinal Products for Veterinary use (CVMP). Guideline on statistical principles for clinical trials for veterinary medicinal products (pharmaceuticals). EMA/CVMP/EWP/81976/2010. London: European Medicine Agency; 2012.

27. Guideline for the testing and evaluation of the efficacy of antiparasitic substances for the treatment and prevention of tick and flea infestation in dogs and cats. 14 July 2016 EMEA/CVMP/EWP/005/2000-Rev.3 Committee for Medicinal Products for Veterinary Use (CVMP). https://www.ema.europa. eu/documents/scientific-guideline/guideline-testing-evaluation-efficacyantiparasitic-substances-treatment-prevention-tick-flea_en-0.pdf.

28. Ripley B, Venables B, Bates DM, Hornik K, Gebhardt A, Firth D. MASS: Support Functions and Datasets for Venables and Ripley's MASS; 2017. https://cran.r-project.org/web/packages/MASS/index.html.

29. Therneau TM. Lumley T. survival: Survival analysis; 2017. https://cran.rproject.org/web/packages/survival/index.html

30. Cuhadar S, Koseoglu M, Atay A, Dirican A. The effect of storage time and freeze-thaw cycles on the stability of serum samples. Biochem Medica. 2013;23:70-7.

31. Walther FM, Allan MJ, Roepke RK, Nuernberger MC. The effect of food on the pharmacokinetics of oral fluralaner in dogs. Parasit Vectors. 2014;7:84.

32. Maroli M, Cianchi T, Bianchi R, Khoury C. Testing insecticide susceptibility of Phlebotomus perniciosus and P. papatasi (Diptera: Psychodidae) in Italy. Ann Ist Super Sanita. 2002;38:419-23.

Ready to submit your research? Choose BMC and benefit from:

- fast, convenient online submission

- thorough peer review by experienced researchers in your field

- rapid publication on acceptance

- support for research data, including large and complex data types

- gold Open Access which fosters wider collaboration and increased citations

- maximum visibility for your research: over $100 \mathrm{M}$ website views per year

At $\mathrm{BMC}$, research is always in progress.

Learn more biomedcentral.com/submissions 